\title{
Application de la théorie des problèmes inverses à l'estimation des paramètres des modèles rhéologiques
}

\author{
Application of the inverse problem theory \\ to rheological model parameter estimation
}

\author{
J.F. SHAO, A. DAHOU, J.P. HENRY \\ Laboratoire de Mécanique de Lille - EUDIL \\ CNRS - GRECO Géomatériaux*
}

Rev. Franç. Géotech. n 57, pp. 75-80 (octobre 1991)

\section{Résumé}

Nous présentons dans cette étude une méthode d'assistance à la détermination des paramètres des modèles rhéologiques. Cette méthode est basée sur une théorie probabiliste des problèmes inverses (TARANTOLA, 1987). Elle tient compte des incertitudes des mesures au Laboratoire et des connaissances a priori des paramètres des modèles rhéologiques. La méthode présentée donne un algorithme général qui permet de déterminer les paramètres des modèles rhéologiques sophistiqués à partir des essais multiaxiaux non conventionnels, voire des essais non homogènes.

\begin{abstract}
In this study we present an assistance method for parameter determination of rheological models. This method is based on a probabilist theory of inverse problem. It takes into account the incertitude degree of experimental results and the a priori knoweldge of model parameters. The present paper gives a general algorithm that allows to determine the model parameters from non conventional multiaxial tests, or even from non homogeneous tests.
\end{abstract}




\section{INTRODUCTION}

La modélisation du comportement complexe des géomatériaux devient le problème majeur dans l'analyse et la conception des ouvrages en génie civil, en génie pétrolier et d'autres constructions souterraines. Le progrès sans cesse de l'informatique rend désormais possible l'utilisation des lois rhéologiques sophistiquées pour décrire le comportement des géomatériaux. De nombreux modèles pour différents types de matériaux ont été développés pendant les deux dernières décennies, LADE (1977), DESAI et SALAMI (1987), DARVE (1978), VALANIS et READ (1982), NOVA et HUECKEL (1982)... Si ces modèles décrivent assez correctement les principaux phénomènes physiques du comportement des matériaux, l'estimation, à partir des essais au Laboratoire, d'un nombre assez important de paramètres introduits dans ces modèles, est souvent une tâche difficile. En général, ces paramètres sont calés à partir des essais au Laboratoire définis a priori avec une méthode artisanale comme la technique graphique, ou avec une méthode numérique simple comme les moindres carrés.

Dans cette étude, nous présentons une approche probabiliste pour la détermination des paramètres des modèles rhéologiques sophistiqués en utilisant une théorie des problèmes inverses, (TARANTOLA, 1987). Dans cette démarche, l'estimation des paramètres est considérée comme un problème inverse. lequel consiste à maximiser l'intersection de deux fonctions de densité de probabilité de type gaussien. La méthode tient compte des incertitudes des mesures expérimentales et des connaissances que l'on peut avoir a priori sur les paramètres des modèles. Elle permet en principe d'estimer les paramètres à partir des essais qui utilisent un chemin de sollicitation quelconque même non homogènes.

Bien que le principe de la méthode présentée soit tout à fait général, il est toutefois nécessaire de souligner que les paramètres d'un modèle rhéologique peuvent en général être distingués en deux types :

- les paramètres a directs "qui peuvent être déterminés à partir des mesures directes au laboratoire comme les paramètres d'élasticité ;

- les paramètres « cachées » dont la détermination à partir des résultats expérimentaux nécessite des dépouillements intermédiaires difficiles comme par exemple les paramètres d'écrouissage.

Il est évident que la méthode inverse est d'autant plus intéressante que la procédure directe de détermination des paramètres est compliquée.

\section{FORMULATION DU PROBLÈME}

\subsection{Définition du problème}

Dans la modélisation du comportement des matériaux, la simulation numérique d'essais effectués au Laboratoire, à l'aide d'un modèle, constitue un problème direct qui peut être décrit par la relation suivante :

$$
D=F(P, C, S)
$$

où $\mathrm{F}$, la fonctionnelle, représente la loi de comportement, C l'ensemble des constantes du problème, S le chemin de sollicitations. D est la réponse du matériau décrite par le modèle, et $\mathrm{P}$ le vecteur des paramètres.

La détermination des paramètres consiste à trouver $\mathrm{P}$ à partir des mesures expérimentales de $\mathrm{D}$, notées $D_{m}$. Ceci revient à définir un problème inverse tel que :

$$
P=F^{-1}\left(D_{m}, C, S\right)
$$

La résolution explicite de ce problème inverse est en général impossible. Il est nécessaire d'utiliser un processus d'optimisation. Le problème consiste donc à déterminer un vecteur $\mathrm{P}$ tel que:

$$
\mathrm{D}_{c}=\mathrm{F}(\mathrm{P}, \mathrm{C}, \mathrm{S})
$$

et l'on obtient :

$$
\underset{\mathrm{Pin}}{\mathrm{M}}\left\|\mathrm{D}_{\mathrm{m}}-\mathrm{D}_{\mathrm{c}}\right\|
$$

où $\mathrm{D}_{\mathrm{c}}$ est le vecteur des réponses calculé par le modèle.

\subsection{Approche statistique}

Afin de résoudre le problème inverse par un processus d'optimisation, nous allons utiliser une approche statistique.

Les mesures expérimentales sont effectuées avec un certain degré d'incertitude. Nous considérons donc que les valeurs expérimentales utilisées dans le problème (2) sont caractérisées par une moyenne $D_{m}$ et les écarts-types $S_{1}$. Ainsi $D_{c}$ est donc considéré comme une variable aléatoire.

Nous faisons l'hypothèse que la variable aléatoire vérifie la distribution de Gauss. Avec cette hypothèse, nous définissons la fonction de densité de probabilité suivante :

$$
\begin{aligned}
& f_{1}(P)=P_{1}=\text { constante } \cdot \exp \\
& \left(-\frac{1}{2}\left[{ }^{t}\left(D_{c}-D_{m}\right) C_{d}^{-1}\left(D_{c}-D_{m}\right)\right]\right)
\end{aligned}
$$

Dans cette relation, $C_{d}$ est la matrice de variancecovariance calculée à partir des écart-types par :

$$
C_{d}=\left[\begin{array}{llll}
S_{1}^{2}(1) & 0 & \bullet & 0 \\
0 & S_{1}^{2}(2) & \bullet & 0 \\
\bullet & \bullet & \bullet & \bullet \\
0 & 0 & \bullet & S_{1}^{2}(n)
\end{array}\right]
$$

où $\mathrm{n}$ est le nombre de mesures expérimentales. Etant donné que $\mathrm{D}_{\mathrm{c}}$ est fonction de $\mathrm{P}$, le problème est donc de trouver $\mathrm{P}$ tel que $\mathrm{D}_{c}$ donne une valeur maximale pour $P_{1}$. Ceci revient alors à minimiser la quantité $\mathrm{S}_{\mathrm{d}}$ :

$$
\mathrm{S}_{\mathrm{d}}={ }^{\mathrm{t}}\left(\mathrm{D}_{\mathrm{c}}-\mathrm{D}_{\mathrm{m}}\right) \mathrm{C}_{\mathrm{d}}^{-1}\left(\mathrm{D}_{\mathrm{c}}-\mathrm{D}_{\mathrm{m}}\right)
$$

La résolution de ce problème de minimisation donne parfois plusieurs solutions possibles, comme ce qui est suggéré par la figure 1. Comme les paramètres d'un modèle de comportement représentent en général une certaine signification physique avec une certaine marge 


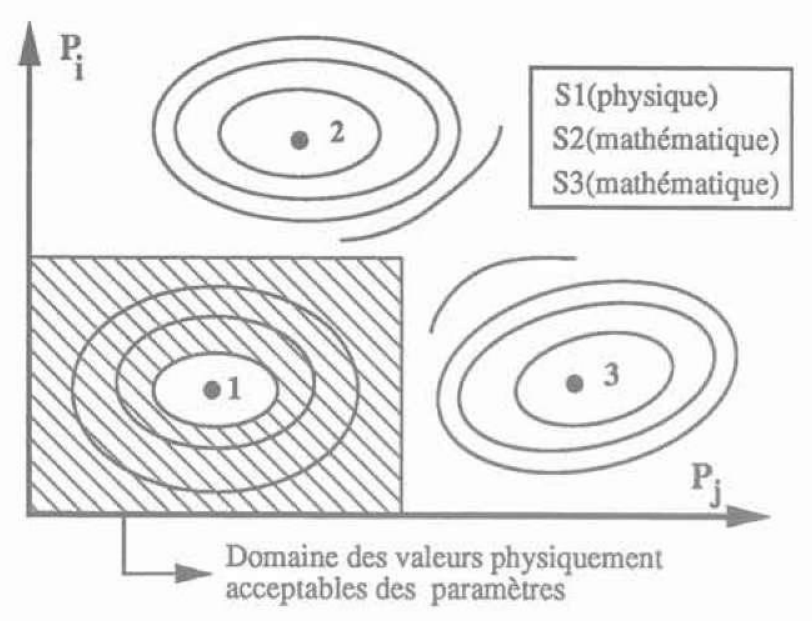

Fig. 1. - Domaine physique des paramètres. Fig. 1. - Physical domain of parameters.

de valeurs physiquement acceptables, il est nécessaire d'éliminer des solutions qui sont purement mathématiques. Ceci nous amène à définir un domaine physique des paramètres à partir des connaissances a priori obtenues par l'étude théorique du modèle ou l'étude paramétrique.

Pour ce fait, nous considérons les valeurs initiales (ou de référence) des paramètres comme la moyenne $\mathrm{P}_{\text {o }}$. La marge physique des paramètres est exprimée par les écarts-types $\mathrm{S}_{2}$. En utilisant de nouveau la distribution de Gauss, on obtient la fonction de densité de probabilité suivante:

$$
\begin{aligned}
& \mathrm{f}_{2}(\mathrm{P})=\mathrm{P}_{2}=\text { constante } \cdot \exp \\
& \left(-\frac{1}{2}\left[{ }^{t}\left(\mathrm{P}-\mathrm{P}_{\mathrm{o}}\right) \mathrm{C}_{\mathrm{p}}^{-1}\left(\mathrm{P}-\mathrm{P}_{\mathrm{o}}\right)\right]\right)
\end{aligned}
$$

Dans cette relation, $\mathrm{C}_{\mathrm{p}}$ est la matrice de variancecovariance pour les paramètres définie par:

$$
\mathrm{C}_{\mathrm{p}}=\left[\begin{array}{llll}
\mathrm{S}_{2}^{2}(1) & 0 & \bullet & 0 \\
0 & \mathrm{~S}_{2}^{2}(2) & \bullet & 0 \\
\bullet & \bullet & \bullet & \bullet \\
0 & 0 & \bullet & \mathrm{S}_{2}^{2}(\mathrm{r})
\end{array}\right]
$$

où $r$ est le nombre des paramètres.

La résolution du problème inverse (2) consiste alors à trouver le vecteur $\mathrm{P}$ dans le domaine physique satisfaisant aux conditions (3) et (4). Ceci correspond à définir l'intersection de $P_{1}$ et $P_{2}$, donc une fonction globale de densité de probabilité.

$$
f(P)=P_{1} P_{2}=\text { constante } \cdot \exp (-\mathrm{S})
$$
avec :

$$
\begin{aligned}
& S=\frac{1}{2}\left[{ }^{t}\left(D_{c}-D_{m}\right) C_{d}^{-1}\left(D_{c}-D_{m}\right)\right. \\
& \left.+{ }^{t}\left(P-P_{o}\right) C_{p}^{-1}\left(P-P_{o}\right)\right]
\end{aligned}
$$

Ainsi la résolution du problème inverse est transformée en la maximisation de $f(P)$ donc en la minimisation de la quantité $\mathrm{S}$. Le vecteur des paramètres à déterminer est celui qui minimise $\mathrm{S}$.
Il existe différentes méthodes de résolution d'un problème de minimisation (FLETCHER, 1980 ; TARANTOLA, 1987). Pour le problème d'estimation des paramètres, du fait qu'un modèle rhéologique définit une fonction dérivable des paramètres $D_{c}(P)$, la méthode de Gauss-Newton paraît la plus puissante et la plus utilisée. C'est cette méthode qui est retenue dans cette étude. Nous trouverons l'algorithme détaillé de Gauss-Newton dans TARANTOLA (1987) et FLETCHER (1980).

\section{TEST ET APPLICATION}

\subsection{Présentation du modèle}

La méthode présentée précédemment est utilisée à l'estimation des paramètres d'un modèle élastoplastique pour roches poreuses (SHAO, 1990). Nous donnons une brève présentation de ce modèle. Il s'agit d'un modèle à deux mécanismes de déformation plastique avec un comportement élastique non linéaire. Les fonctions de charge et les lois d'écrouissage sont les suivantes:

- pour le mécanisme hydrostatique :

$$
\begin{aligned}
& \mathrm{F}_{\mathrm{c}}=\mathrm{I}_{1}-\mathrm{y}_{\mathrm{c}}=0 \\
& \mathrm{y}_{\mathrm{c}}=\mathrm{y}_{\mathrm{c}}^{0}+\mathrm{a} \mathrm{P}_{\mathrm{a}} \xi_{\mathrm{c}}^{\mathrm{n}} \exp \left(\mathrm{c} . \xi_{\mathrm{c}}\right)
\end{aligned}
$$

- pour le mécanisme déviatorique :

$$
\begin{gathered}
F_{d}=\left(\frac{I_{1}^{3}}{I_{3}}-27\right)\left(\frac{I_{1}}{P_{a}}\right)^{m}-y_{d}=0 \\
y_{d}=y_{d}^{\circ}+\frac{\left(y_{d}^{\mathrm{f}}-y_{d}^{\circ}\right) \xi_{d}}{b+\xi_{d} \gamma}
\end{gathered}
$$

Dans les relations précédentes, $I_{1}, I_{2}$ et $I_{3}$ sont les trois invariants du tenseur de contraintes. $\mathrm{P}_{\mathrm{a}}$ est la pression atmosphérique exprimée dans les mêmes unités que les contraintes afin d'obtenir des paramètres sans dimensions. $\xi_{c}$ et $\xi_{\text {d }}$ définissent les déformations plastiques équivalentes pour les deux mécanismes plastiques et sont utilisés comme variables d'écrouissage du modèle (SHAO et HENRY, 1990). y y et $y_{d}^{\circ}$ représentent les limites d'élasticité initiales du matériau pour les deux mécanismes respectifs et définis par :

$$
\begin{gathered}
y_{c}^{\circ}=3\left(\mathrm{Po}_{\mathrm{c}}+\mathrm{RP}_{\mathrm{a}}\right) \\
y_{\mathrm{d}}^{\circ}=\mathrm{t}\left(\mathrm{P}_{\mathrm{o}}-\sigma_{\mathrm{o}}\right) / \mathrm{Pa}, \mathrm{y}_{\mathrm{d}}^{\circ}>0
\end{gathered}
$$

où $\sigma_{\circ}$ est la contrainte hydrostatique initiale du problème. $\mathrm{P}_{0}$ et $\mathrm{t}$ sont deux paramètres du modèle. $\mathrm{R}$ est le paramètre représentant la cohésion du matériau. En plus, une translation de l'espace des contraintes est proposée afin de prendre en compte la cohésion des roches :

$$
\sigma_{\mathrm{ij}}{ }^{*}=\sigma_{\mathrm{ij}}+\mathrm{RP} \mathrm{P}_{\mathrm{a}} \delta_{\mathrm{ij}}
$$

où $\delta_{\text {ji }}$ est le symbole de Kronecker. Ainsi les invariants utilisés dans le modèle sont calculés avec $\sigma_{i j}{ }^{\circ}$. Dans la relation (15), b varie en fonction de $I_{1}$ pour mieux interpréter la dépendance du comportement d'écrouis- 
sage des roches poreuses de la contrainte hydrostatique :

$$
\mathrm{b}=\ln \left(\alpha+\beta\left(\mathrm{I}_{1} / 3 \mathrm{p}_{\mathrm{a}}\right)\right)
$$

où $\alpha$ et $\beta$ sont deux paramètres du matériau.

Enfin une loi d'élasticité non linéaire est utilisée dans le modèle. Le module de compressibilité $\mathrm{K}$ varie en fonction de $\mathrm{I}_{1}$ et par contre le coefficient de Poisson $\nu$ est considéré comme constant :

$$
\mathrm{K}=\mathrm{K}_{\mathrm{o}}\left(\mathrm{I}_{1} / 3 \mathrm{P}_{\mathrm{o}}\right)^{\mathrm{n}}
$$

où $\mathrm{K}_{\mathrm{o}}$ est la valeur initiale de $\mathrm{K}$.

En résumé, ce modèle comprend 13 paramètres qui sont donnés dans le tableau 1. Parmi ces paramètres $\mathrm{K}_{0}, \nu, \mathrm{P}_{0}, \mathrm{t}, \mathrm{R}, \mathrm{m}$, y $\mathrm{y}_{\mathrm{d}}^{\mathrm{t}}$ sont de type direct, dont peuvent être déterminés à partir des mesures directes. Les paramètres $\mathrm{a}, \mathrm{n}, \mathrm{c}, \alpha, \beta, \gamma$ sont de type caché. Nous présentons par la suite seulement la détermination de ceux-ci ainsi que $\mathrm{m}$ et $\mathrm{y}_{\mathrm{d}}$ car ils interviennent dans la loi d'écrouissange du modèle par la méthode inverse. C'est sur ce type de paramètres que l'on montrera la performance de la méthode.

Tableau 1. - Paramètres du modèle.

Table 1. - Model parameters.

\begin{tabular}{|l|c|}
\hline Paramètres d'élasticité & $\mathrm{K}_{0}, \nu$ \\
\hline Paramètres pour les limites d'élasticité & $\mathrm{P}_{\mathrm{o}}, \mathrm{t}$ \\
\hline Paramètres de cohésion & $\mathrm{R}$ \\
\hline Paramètres à la rupture & $\mathrm{m}, \mathrm{y}_{\mathrm{d}}^{\mathrm{r}}$ \\
\hline Paramètres d'écrouissage hydrostatique & $\mathrm{a}, \mathrm{n}, \mathrm{c}$ \\
\hline Paramètres d'écrouissage déviatoire & $\alpha, \beta, \gamma$ \\
\hline
\end{tabular}

Avant d'aborder l'application de la méthode d'inversion à la détermination de ces paramètres, il est utile de préciser le contenu des variables annoncées dans la section précédente. Un modèle de comportement prévoit la réponse d'un matériau soumis à une certaine sollicitation. Cette relation réponse-sollicitation définit la fonctionnelle $\mathrm{F}$ dont la forme explicite est en général impossible à donner. La réponse est conditionnée par le vecteur $\mathrm{P}$ qui contient 13 composantes pour le modèle retenu. Le vecteur $\mathrm{C}$ précise les constantes du problème, telles que le type de chemins de sollicitation (triaxial, œdométrique, drainé, non drainé, etc.) et le nombre d'incréments par exemple. Le contenu des vecteurs de la sollicitation $\mathrm{S}$ et de la réponse $D_{c}$ dépend du chemin de sollicitation. Pour un essai triaxial drainé à contrainte axiale imposée, $\mathrm{S}$ est composé des incréments de la contrainte axiale et $\mathrm{D}_{c}$ des incréments de la déformation axiale et celle volumique correspondantes. S'il existe des essais répétés, il sera possible de calculer une moyenne des valeurs expérimentales de ces déformations et des écarts-types associés qui constituent les vecteurs $D_{m}$ et $S_{1}$. Enfin, des connaissances a priori sur le domaine des valeurs physiquement acceptables pour les paramètres permettent de déterminer les vecteurs $\mathrm{P}_{0}$ et $\mathrm{S}_{2}$. Par exemple on peut utiliser pour $\mathrm{P}_{0}$ les valeurs des paramètres utilisées pour un matériau dont les caractéristiques physiques (la porosité par exemple) sont relativement proches de celles du matériau à étudier.

\subsection{Tests de la méthode}

L'objectif est de tester l'efficacité de la méthode. Le principe de base de ces tests est de créer des courbes de comportement théorique à partir de valeurs imposées des paramètres au modèle. Ensuite, nous prendrons les résultats de la simulation comme la moyenne des résultats expérimentaux artificiels $D_{m}$ et nous introduirons des perturbations sur certains des paramètres. Les valeurs perturbées de ces paramètres seront donc utilisées comme $\mathrm{P}_{0}$. Enfin, nous utilisons la méthode inverse pour retrouver les valeurs réelles des paramètres perturbés. Dans les tableaux 2 et 3 , deux exemples de tests sont présentés. Nous pouvons constater que la méthode inverse donne les valeurs des paramètres avec une bonne précision. Il est clair que la convergence de la méthode sera moins rapide lorsque les valeurs perturbées des paramètres sont plus loin de leurs valeurs réelles.

Tableau 2. - Test sur le paramètre d'écrouissage a avec un essai hydrostatique.

Table 2, - Test on the yielding parameter a with a hydrostatic test.

\begin{tabular}{|l|c|}
\hline Valeur réelle & 1800 \\
\hline Valeur perturbée & 1500 \\
\hline Solution inverse & 1796.92 \\
\hline Nombre d'itérations & 4 \\
\hline
\end{tabular}

Tableau 3. - Test sur les paramètres de rupture $m, y_{d}^{\prime}$ avec un essai triaxial.

Table 3. - Test on the failure parameters $m, y_{d}^{r}$ with a triaxial test.

\begin{tabular}{|l|c|c|}
\hline \multicolumn{1}{|c|}{ Paramètres } & $\mathrm{m}$ & $\mathrm{y}_{\mathrm{d}}^{\mathrm{r}}$ \\
\hline Valeurs réelles & 0,18 & 59 \\
\hline Valeurs perturbées & 0,15 & 70 \\
\hline Solutions inverses & 0,178 & 58,22 \\
\hline Nombre d'itérations & \multicolumn{2}{|c|}{14} \\
\hline
\end{tabular}

\subsection{Applications}

Nous présentons deux exemples de détermination des paramètres du modèle élastoplastique choisi pour deux roches très poreuses (craies). Le premier exemple est sur la craie présentée dans la référence (SHAO, 1990 a) (craie blanche $40 \%$ de porosité). Après l'étude théorique et paramétrique du modèle (méthode directe), nous avons utilisé les moyennes et les écarts-types suivants comme information a priori sur les paramètres $\mathrm{a}, \mathrm{n}, \mathrm{c}, \mathrm{m}, \mathrm{y}_{\mathrm{d}}^{\mathrm{r}}, \alpha, \beta, \gamma$ (tableau 4). 
Tableau 4. - Valeurs initiales et écarts-types des paramètres.

Table 4. - Initial values and standard deviation of parameters.

\begin{tabular}{|c|c|c|}
\hline \multirow{2}{*}{$a$} & $\mathrm{P}_{\mathrm{o}}$ & $\mathrm{S}_{2}$ \\
\cline { 2 - 3 } $\mathrm{n}$ & 1350 & 150 \\
$\mathrm{c}$ & 0,41 & 0,137 \\
$\mathrm{~m}$ & 15 & 5 \\
$\mathrm{y}_{d}^{r}$ & 0,15 & 0,049 \\
$\alpha$ & 60 & 13,3 \\
$\beta$ & 0,975 & 0,007 \\
$\gamma$ & $0,5 \times 10^{-3}$ & $0,15 \times 10^{-3}$ \\
\hline
\end{tabular}

Quant aux résultats expérimentaux utilisés, le manque d'essais répétés ne permet pas d'évaluer précisément les moyennes $D_{m}$ et les écarts-types $S_{1}$. Des valeurs arbitraires correspondant à $10 \%$ d'erreurs relatives à la courbe moyenne sont introduites pour ce premier exemple comme d'ailleurs pour le deuxième.

Ce premier exemple montre la détermination des trois paramètres d'écrouissage hydrostatique a, n, c à partir d'un essai de compression isotrope, ainsi que les autres paramètres à partir des essais triaxiaux. Les valeurs de ces paramètres sont données dans le tableau 5. Dans les figures 2 et 3 sont présentées les courbes comparatives de simulations numériques et expérimentales. Nous remarquons la bonne concordance entre les résultats.

Tableau 5. - Valeurs des paramètres pour l'exemple 1. Table 5. - Values of the parameters for the example 1.

\begin{tabular}{|c|c|}
\hline $\mathrm{a}$ & 1078 \\
$\mathrm{n}$ & 0,265 \\
$\mathrm{c}$ & 10,40 \\
$\mathrm{~m}$ & 0,162 \\
$\mathrm{yd}_{\mathrm{r}}^{\mathrm{r}}$ & 59,04 \\
$\alpha$ & 0,966 \\
$\beta$ & $5,75 \times 10^{-4}$ \\
$\gamma$ & 0,95 \\
\hline
\end{tabular}

Le deuxième exemple consiste à déterminer les paramètres retenus à partir d'un essai œdométrique sur une autre craie blanche (craie de $45 \%$ de porosité - SHAO, 1990 b). L'essai œdométrique est l'essai le plus couramment utilisé en génie pétrolier. Il a le désavantage de ne pouvoir être utilisé avec les méthodes directes pour déterminer les paramètres des modèles rhéologiques. Cependant, en utilisant la technique d'inversion présentée ici, les paramètres peuvent être calculés sans difficultés (tableau 6).

Les comparaisons entre la simulation numérique obtenue à partir du tableau 6 et l'expérience sont présentées dans les figures 4 et 5 . Nous constatons également de bonnes corrélations qui permettent de conclure d'une part à la qualité du modèle, et d'autres part à la bonne qualité de détermination des paramètres. Il est utile de signaler que la qualité des paramètres obtenus par la présente méthode est liée directement à la qualité de la moyenne $\mathrm{D}_{\mathrm{m}}$ et des écarts-

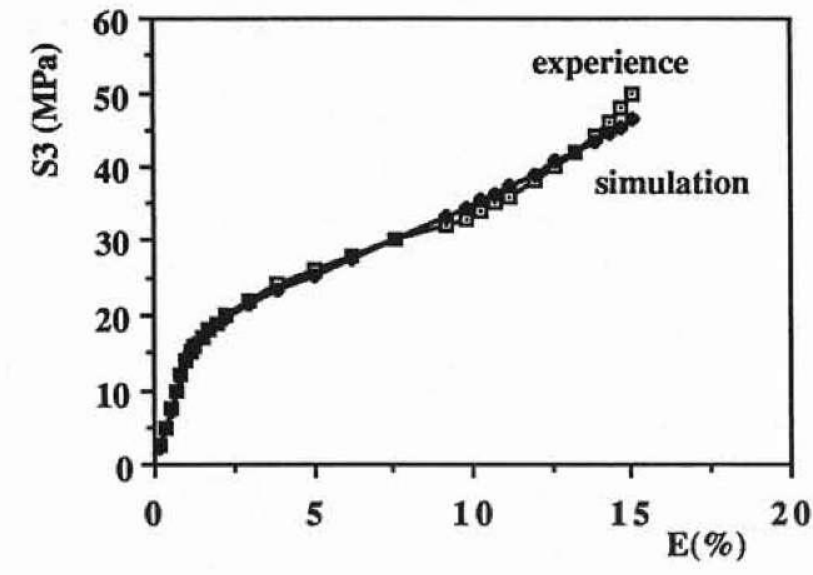

Fig. 2. - Comparaison entre l'expérience et la simulation pour l'essai hydrostatique.

Fig. 2. - Comparison between the experiment and the simulation for the hydrostatic test.

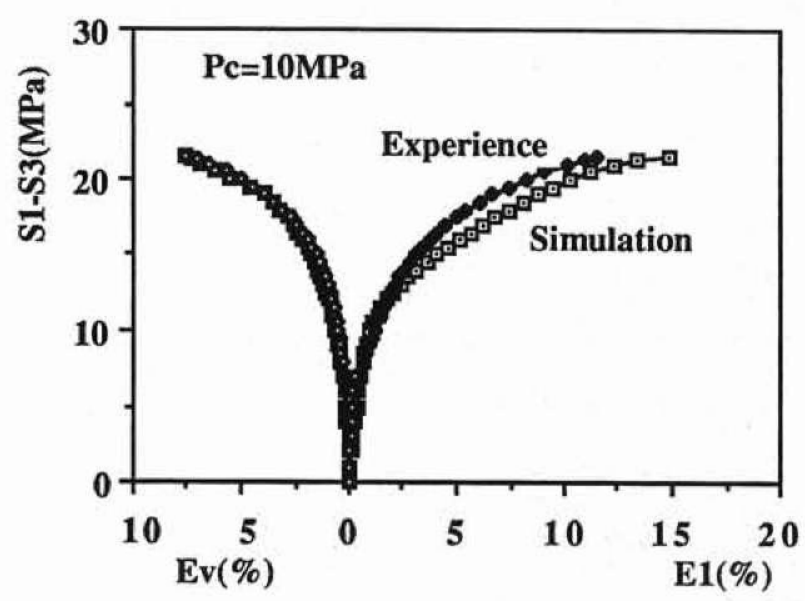

Fig. 3. - Comparaison entre l'expérience et la simulation pour l'essai triaxial.

Fig. 3. - Comparison between the experiment and the simulation for the triaxial test.

Tableau 6. - Valeurs des paramètres pour l'exemple 2. Table 6. - Values of the parameters for the example 2.

\begin{tabular}{|c|c|}
\hline $\mathrm{a}$ & 1897 \\
$\mathrm{n}$ & 0,435 \\
$\mathrm{c}$ & 27,27 \\
$\mathrm{~m}$ & 0,245 \\
$\mathrm{y}_{\mathrm{d}}^{\mathrm{r}}$ & 56,29 \\
$\alpha$ & 0,99 \\
$\beta$ & $7,6 \times 10^{-4}$ \\
$\gamma$ & 0,934 \\
\hline
\end{tabular}

types $S_{1}$ pour les résultats expérimentaux. Au point de vue statistique, cette qualité s'améliore lorsque le nombre d'essais répétés augmente. De ce fait, la méthode d'inversion fournit les valeurs les plus probables des paramètres.

\section{CONCLUSION ET PERSPECTIVES}

Une méthode basée sur la théorie des problèmes inverses pour l'estimation des paramètres des lois 


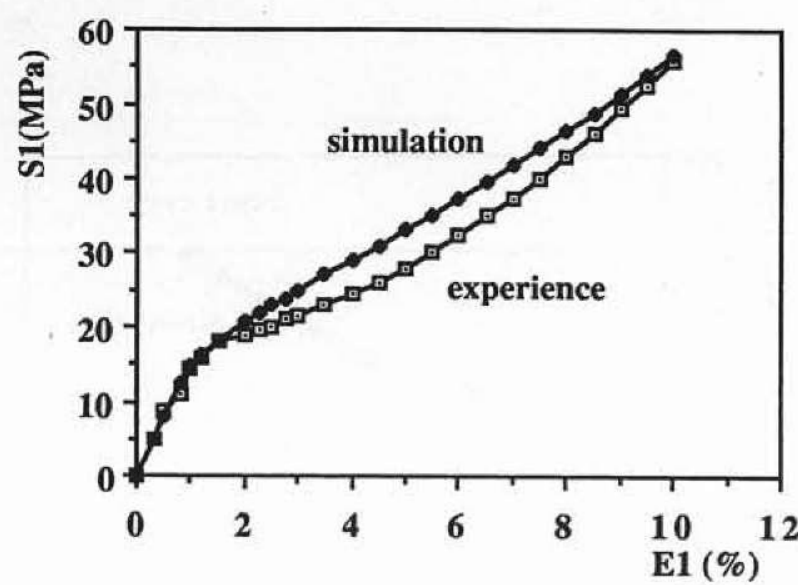

Fig. 4. - Comparaison entre l'expérience et la simulation pour l'essai œedométrique (contrainte axiale).

Fig. 4. - Comparisons between the experiment and the simulation for the œedometric test (axial stress).

rhéologiques vient donc d'être présentée. Elle considère le problème d'estimation des paramètres comme un problème inverse. La formulation d'un problème d'optimisation qui donne la solution du problème inverse est basée sur une approche statistique. La méthode présentée permet d'estimer les paramètres à partir d'un chemin de sollicitation quelconque dans lequel les paramètres en question sont activés. Les tests et les exemples d'application présentés montrent la performance prometteuse de la méthode. Il est toutefois nécessaire de rappeler que le problème d'une bonne estimation des paramètres des modèles rhéologiques est très complexe et nécessite une bonne connaissance du problème direct. La méthode présentée fournit un outil supplémentaire intéressant, mais ne prétend nullement être universelle. La démarche probabiliste et l'applicabilité à des essais non conventionnels représentent les particularités de cette méthode.

Il est possible de généraliser cette méthode pour déterminer les paramètres à partir d'essais non homogènes, voire d'essais in situ. Enfin, la méthode peut être également un outil intéressant pour étudier la stabilité des modèles par rapport à l'interdépendance des paramètres.

\section{BIBLIOGRAPHIE}

[1] CHARLEZ Ph. (1990), Application des problèmes inverses à la mécanique des roches. Communication personnelle.

[2] DARVE F. (1978), Une formulation incrémentale des lois rhéologiques. Applications aux sols. Thèse de Doctorat d'Etat, Université de Grenoble.

[3] DEANATAL J.S. (1987), Computer-aided calibration of constitutive models. Constitutive laws for Engineering materials, Theory and applications, Desai et al. (eds), Elsevier, pp. 1181-1188.

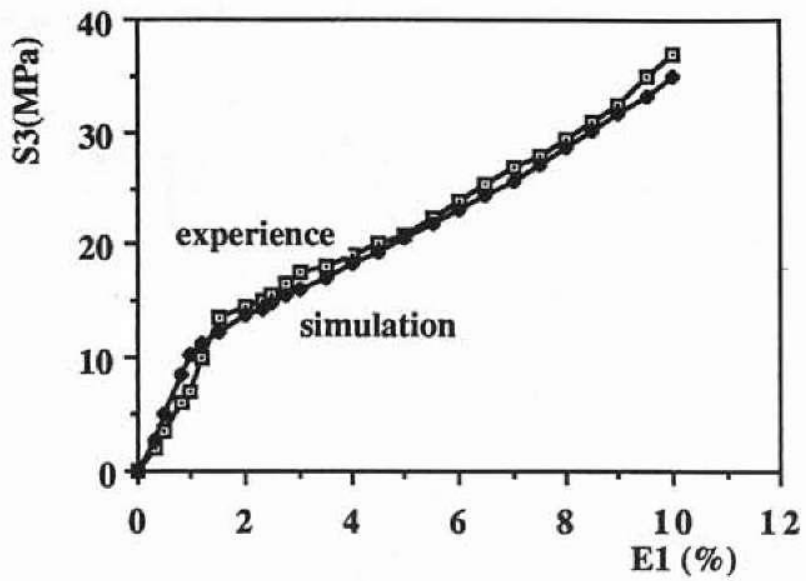

Fig. 5. - Comparaison entre l'expérience et la simulation pour l'essai œdométrique (contrainte latérale).

Fig. 5. - Comparisons between the experiment

and the simulation for the œdometric test (lateral stress).

[4] DESAI C.S., SALAMI M.R. (1987), A constituitive model and associated testing for soft rock. Int. J. Rock Mech. Min. Sci. \& Geomech. Abstr. 24, n 5, pp. 299-307.

[5] FLETCHER R. (1980), Practical methods of optimization. John Wiley and sons.

[6] HUMPHREY D.N., HOLTZ R.D. (1987), A procedure to determine cap model parameters. Constituitive laws for Engineering materials, Theory and applications, Desai et al. (eds), Elsevier, pp. 1225-1232.

[7] JU J.W., SIMO J.C., PISTER K.S., TAYLOR R.L. (1987), A parameters estimation algorithm for inelastic material model. Constituitive laws for engineering materials. Theory and applications, Desai et al. (eds), Elservier, p. 1233-1238.

[8] LADE P.V. (1977), Elastoplastic stress-strain theory for cohesionless soil with curved yield surfaces. Int. J. Solids Structures, vol. 13, pp. 1019-1035.

[9] NOVA R., HUECKEL T. (1982), A model for soil behaviour in plastic and hysteretic ranges. Proc. of the international workshop on Constituitive relations for soils, Grenoble, pp. 289-330.

[10] SHAO J.F., HENRY J.P. (1990 a), Validation of an elastoplastic model for chalk. Computers and Geotehcnics, 9, pp. 257-272.

[11] SHAO J.F., HENRY J.P. (1990 b), Constitutive modelling of chalk behaviour. Third North Sea Chalk Symposium, Copenhagen.

[12] TARANTOLA A. (1987), Inverse problem theory. Elsevier.

[13] VALANIS K.C., READ H.G. (1982), A new endochronic plasticity model for soils. Soil mechanics Transient and cyclic loads, Pande et al. (eds), John Willey, pp. 375-417.

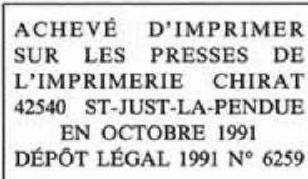

IMPRIMÉ EN FRANCE 